\title{
Comunicación de un caso de cisticercosis subcutánea
}

\author{
Sylvia Vidal
}

\section{Report of case of subcutaneous cysticercosis}

Infection of the larval form (cysticerco) of Taenia in any tissue or organ is known as the disease cysticercosis. Many sites of infection have been documented but the central nervous system has been the most common. It present a case report of a 19 years old patient with a subcutaneous cysticercosis confirmed with biopsy.

Key words: Cysticercosis, Taenia, disease.

Palabras clave: Cisticercosis, Taenia solium, cisticerco.

\section{Introducción}

$T$ aenia solium se conoce como "solitaria" y desde la antigüedad se reconoce como una infección del ser humano. Es un platelminto, perteneciente a la subclase Eucestoda, orden Cyclophyllidea y familia Taeniidae. El parásito es hermafrodita y tiene dos tipos de huéspedes: uno definitivo que aloja al estado adulto o tenia (el humano) y otro intermediario que aloja al estado larval o cisticerco (el cerdo y el hombre). En el humano causa "teniasis" cuando la fase adulta de Taenia solium se establece en el intestino y "cisticercosis" cuando la fase larvaria se encuentra en tejidos extraintestinales. En el cerdo se produce sólo cisticercosis ${ }^{1,2}$.

El consumo de huevos de Taenia solium por el ser humano, puede causar cisticercosis en diferentes tejidos y una infección grave llamada neurocisticercosis cuando afecta al sistema nervioso central. La infección de otros órganos es raramente detectada ${ }^{1,2}$.

La cisticercosis subcutánea es asintomática, pero al estar presente es de gran ayuda para el clínico, ya que permite detectarla al examen físico y confirmar el diagnóstico mediante una biopsia ${ }^{3,4}$.

\section{Caso clínico}

Se presenta el caso clínico de una mujer de 19 años proveniente de sector rural de Huaquén, perteneciente a la comuna de Curepto de la Región del Maule. Consultó en un centro privado por presentar una lesión de piel con aspecto de una protuberancia blanda, sin signos inflamatorios, en la nariz y espalda, con un diámetro entre 0,5 y $0,7 \mathrm{~cm}$.

Se realizó escisión de las lesiones a las que se les realizó una biopsia, que concluyó que se trataba de cisticercos.

Se realizó un TAC cerebral que resultó normal, descartando una neurocisticercosis. Posteriormente la paciente y su familia fueron derivados a Talca para realizar un estudio parasitológico al grupo familiar.

A todos los integrantes de la familia: dos adultos, la paciente y una niña de 9 años, se les realizaron un parasitológico seriado de deposiciones (PSD) por el método PAFS. Todos resultaron negativos para huevos de $T$. solium, encontrándose sólo parásitos comensales en uno de los adultos (quistes de Iodamoeba butschlii). También se realizó un estudio serológico para la búsqueda de IgG mediante la técnica de ELISA para cisticercosis (Diagnostic Automatition $({ }$, Inc), que ocupa como antígeno fluido del quiste de Taenia solium (Cisticercos cellulosae) cuyos resultados también fueron negativos en todos los integrantes: 0,02 a 0,03 U.D.O) (valor de referencia: 0,0 a 0,3 U.D.O.)

\section{Discusión}

Los huevos de T. solium pueden ser ingeridos desde el suelo, agua o alimentos contaminados con heces humanas o desde las manos de un portador de una tenia adulta o de alguien de su ambiente cercano. Luego de ser ingeridos, la envoltura de los huevos es disuelta. Los embriones liberados en el intestino delgado atraviesan la mucosa intestinal activamente, llegando al torrente sanguíneo y transportados a los diversos tejidos del organismo ${ }^{1-4}$.

Al parecer la infección puede establecerse en los diferentes tejidos pero sobrevive por mayor tiempo en lugares inmunológicamente protegidos, como el sistema nervioso central (SNC) o el globo ocular.

El hombre es el único huésped que puede alojar a la Taenia solium adulta, por lo tanto es la única fuente de infección de la cisticercosis, siendo posible desarrollar la cisticercosis por autoinfección, aunque esto es poco frecuente. Las personas que viven en el mismo hogar del portador de la tenia tienen un mayor riesgo de contraer cisticercosis que otras personas.
Universidad de Talca, Chile. Laboratorio de Parasitología.

No hay conflictos de intereses. Fuente de financiamiento: Departamento de Microbiología. Universidad de Talca.

Recibido: 5 de mayo de 2012 Aceptado: 22 de abril de 2013 
El hombre adquiere la teniasis al comer carne de cerdo mal cocida con cisticercos. Los cerdos se infectan al ingerir huevos de tenia excretados en las heces del portador humano.

La infección con la forma larvaria o cisticercosis es un problema de salud pública en la mayor parte del mundo. En los E.U.A., la cisticercosis es considerada una infección desatendida asociada a la pobreza, ya que se presenta en personas con escasos recursos y acceso limitado a la atención médica.

La cisticercosis es endémica en todos los continentes, excepto en Australia y es muy infrecuente en países musulmanes donde está prohibido comer carne de cerdo. Es común en el África subsahariana, China, el subcontinente indio y el sureste asiático y es altamente endémica en áreas rurales de América Latina como México, Guatemala, El Salvador, Honduras, Colombia, Ecuador, Perú, Bolivia y Brasil. Sin embargo, la verdadera incidencia de cisticercosis en humanos y porcinos es desconocida.

La neurocisticercosis afecta hombres y mujeres de todas las edades, con un peak de incidencia entre los 30 y 50 años de edad. Es uno de los mayores problemas de salud pública, esto visto en su prevalencia, que puede alcanzar hasta el 3,6\% de la población general en algunas regiones.

Además del SNC, los cisticercos también pueden localizarse en el tejido subcutáneo, ocular y musculatura esquelética. La cisticercosis subcutánea representa sólo alrededor del $0,9 \%$ de todos los casos de cisticercosis humana ${ }^{1-4}$.

La afectación musculo-cutánea, no suele producir limitación funcional ni molestias. Los pacientes si consultan, suele ser por la presencia de nódulos subcutáneos no dolorosos.

El control de cisticercosis en áreas endémicas depende principalmente del mejoramiento en el saneamiento e higiene personal de la población en riesgo. Un enfoque reciente más específico ha sido disminuir la prevalencia de infestación por $T$. solium mediante el tratamiento masivo de poblaciones con dosis tenicidas de praziquantel o niclosamida y de esta manera reducir el número de casos de cisticercosis en humanos y en cerdos, rompiendo la cadena de transmisión ${ }^{5-7}$. Sin embargo, se debe actuar con precaución ya que los fármacos utilizados no son ovicidas.

En áreas no-endémicas, los miembros de un grupo familiar y otros contactos cercanos con una historia de viaje a zonas endémicas, deben ser investigados para descartar la presencia de tenias intestinales y tratados rápidamente, ya que los portadores de tenias tienen un mayor riesgo de cisticercosis como resultado de la autoinfección, y frecuentemente sirven como una fuente de diseminación de cisticercosis a otros contactos. Para la detección de portadores de tenias intestinales, se puede investigar la eliminación de proglótidas en las heces, mediante técnicas parasitológicas apropiadas ${ }^{8,9}$. Se debe recomendar también la realización de TAC cerebrales en aquellos individuos seropositivos, para descartar el compromiso del $\mathrm{SNC}^{10}$.

Los manipuladores de alimentos en áreas endémicas deberían ser sometidos a diagnóstico parasitológico y/o tratamiento empírico con drogas tenicidas ${ }^{14-17}$.

El periodo entre la infección inicial y la aparición de los síntomas es muy variable; éste puede ser de algunos meses o de varios años.

En los países latinoamericanos la ubicación principal de los cisticercos es el SNC. La expresión clínica de la cisticercosis es polimorfa; la enfermedad puede ser desde asintomática hasta incapacitante y en ocasiones mortal. El cuadro clínico depende de la localización de la cisticercosis. Cuando afecta al SNC las manifestaciones dependen del número, localización y estado evolutivo del parásito. Las más comunes son epilepsia de inicio tardío y cefalea $^{11}$. Su localización más común es la subaracnoidea, seguida de la parenquimatosa.

Actualmente el diagnóstico se debe apoyar con estudios de imágenes: la tomografía computarizada (TAC) ${ }^{10}$, o resonancia nuclear magnética (RNM). Esta última, es considerada la técnica de elección en la práctica clínica, ya que es más sensible que la TAC para diagnóstico de neurocisticercosis activa. Desafortunadamente estas técnicas de imagen no son accesibles para la mayor parte de la población que padece la enfermedad. Por ello, se están desarrollando pruebas diagnósticas, económicas y prácticas, orientadas a la identificación de anticuerpos contra el cisticerco. La técnica de ELISA es la que actualmente ha mostrado mayor sensibilidad (99\%) y especificidad $(99 \%)^{12,13}$. Si la prueba es utilizada en líquido cefalorraquídeo, existe la certeza de que se trata de neurocisticercosis, pero si se realiza en suero, un resultado positivo no necesariamente indica la enfermedad, sino el contacto con el parásito. Es por ello que se están evaluando ensayos que determinan la presencia del antígeno parasitario y así distinguir entre las infecciones activas y las inactivas o la exposición al parásito.

La tenia adulto es el blanco óptimo de ataque, ya que su desaparición significaría un considerable descenso en la infección; además, es la fase parasitaria más crítica en el ciclo de vida de esta zoonosis y, a la vez, la más vulnerable para establecer las alternativas de prevención y control ${ }^{14}$.

Existe una revisión actualizada sobre aspectos destacados de esta enfermedad, la importancia epidemiológica en Chile, los problemas diagnósticos, la existencia de diferentes tipos de cisticercosis, de diferente pronóstico, las decisiones terapéuticas y el estudio de los familiares ${ }^{18}$.

\section{Resumen}

La infección por la forma larvaria (cisticerco) de Taenia solium en cualquier tejido u órgano se conoce 
como cisticercosis. Existen numerosos reportes de casos, siendo la mayoría de ellos cisticercos en sistema nervioso central. El compromiso de otros órganos es raramente detectado. Se presenta el caso de una mujer de 19 años con una cisticercosis subcutánea que fue confirmada con biopsia.

\section{Referencias bibliográficas}

1.- Botero D. Teniasis-cisticercosis. En: Goldsmith R, Heyneman D, editores Parasitología y Medicina Tropical. México DF: Manual Moderno; 1995. p. 632-46.

2.- Evans C A W, García H H, Gilman R H. Larval cestode infections: Cysticercosis. En: Strickland GT, Ed. Hunter's Tropical Medicine and Emerging Infectious Diseases. Philadelphia: W.B.Saunders; 1999: 862-5.

3.- Náquira C. Taenia solium: biological cycle and characteristics. En: García HH, Martinez SM. Taenia solium. Taeniasis/Cysticercosis. $2^{\text {da }}$. edición.

Lima: Ed. Universo; 1999: 7-14.

4.- Pawlowski Z, Allan J, Sarti E. Control of Taenia solium taeniasis / cysticercosis: from research towards implementation. Int J Parasitol 2005; 35: 1221-32.

5.- Corona T, Lugo R, Medina, R, Sotelo J. Singleday praziquantel therapy for neurocysticercosis. N Engl J Med 1996; 334: 125.

6.- Flisser A, Madrazo I, Plancarte A, Schantz P, Allan J, Craig P, et al. Neurological symptoms in occult neurocysticercosis after single taenicidal dose of praziquantel. Lancet 1993; 342: 748.

7.- $\quad$ Nogales-Gaete J, Arriagada C, Salinas R. Tratamiento de la neurocisticercosis: revisión crítica. Rev Med Chile 2006; 134: 789-96.

8.- Allan J C, Ávila G, García Noval J, Flisser A, Craig P S. Immunodiagnosis of taeniasis by coproantigen detection. Parasitology 1990; 101: 473-7.

9.- Mercado R, Muñoz V, Lorca M, García A. Métodos de diagnóstico directo. En: Atías A. Parasitología Médica. Edit. Mediterráneo. Santiago de Chile; 1999, p. 561-570.

10.- Zee C S, Segall H D, Boswell W, Ahmadi J, Nelson M, Colleti P. MR imaging of neurocysticercosis. J Comput Assist Tomogr 1988; 12: 927-34

11.- García HH, Gilman R, Martinez M, Tsang VC, Pilcher JB, Herrera G, et al. Cysticercosis as a major cause of epilepsy in Peru. Lancet 1993; 341: 197-200.

12.- Mondragón A, Plancarte A, Flisser A. El diagnóstico de la cisticercosis humana por ELISA. Salud Pública Méx 1994; 36: 393-8.
13.- Martínez-Maya J J, de Aluja A S, Ávila-Ramírez G, Aguilar-Vega L, Plancarte-Crespo A, Jaramillo-Arango C J, et al. Teniasis y detección de anticisticerco en personas de una comunidad rural del Estado de Guerrero. Salud Pública Méx 2003; 45: 84-9.

14.- Sarti E. La teniosis y cisticercosis por Taenia solium. Salud Pública Méx 1997, 39: 225-231.

15.- Alvarez-Rodríguez E, Torres-Gárate R, Gutiérrez Larráinzar A, Cabello J, Espinós Pérez D. Neurocisticercosis: recomendaciones de tratamiento a propósito de tres casos. An Med Interna 2004; 21: 382-6.

16.- Garcia H H, Del Brutto O H. Neurocysticercosis: updated concepts about an old disease. Lancet Neurol 2005; 4: 653-61.

17.- Saavedra H, Gonzales I, Alvarado M A, Porras M A, Vargas V, Cjuno R A, et al. Diagnóstico y manejo de la neurocisticercosis en el Perú. Rev Peru Med Exp Salud Pública 2010; 27: 586-91.

18.- Fica C A, Castro S M, Soto S A, Flores M C, Oelker B C, Weitzel T. Neurocisticercosis: una enfermedad desatendida en Chile. Rev Chilena Infectol 2012; 29: 72-81. 